\title{
Integrated Adaptive Guidance and Control of Constrained Nonlinear Air-breathing Hypersonic Vehicle Models
}

\author{
Andrea Serrani*, Alicia M. Zinnecker, Lisa Fiorentini, Michael A. Bolender, and David B. Doman
}

\begin{abstract}
This paper presents the design of an adaptive flight control systems for constrained air-breathing hypersonic vehicle models. The proposed architecture comprises a robust adaptive nonlinear inner-loop controller, and a self-optimizing guidance scheme that shapes the reference to be tracked in order to avoid the occurrence of control input saturations. The scheme is explicitly designed to account for the presence of a state-dependent input saturation on the control loop for the vehicle longitudinal velocity, arising from physical limitations in the propulsion system. The approach is based on the integration of a previously-developed adaptive controller with a self-tuning pre-filter which shapes the reference command to maintain the control signal within feasible values. The reference command are left unaltered whenever there is sufficient control authority for stable tracking. Simulation results are provided to show the effectiveness of the method.
\end{abstract}

\section{INTRODUCTION}

Control of hypersonic air-breathing vehicles presents numerous challenges, stemming from the complexity of the dynamics and the unprecedented level of coupling between the airframe and the propulsion system. The longitudinal model of the dynamics of this kind of aircraft are known to be unstable, non-minimum phase with respect to the regulated output, and affected by significant model uncertainty [1]-[4]. Recently, a significant research effort has been spent towards the development of more sophisticated models of hypersonic vehicle dynamics that are amenable to control system design [5], [6], in parallel with the development of control solutions capable to deal with the specific issues inherent to this class of aircraft (see [7]-[11], to cite just a few). In addition to the aforementioned difficulties, the presence of unavoidable constraints on the control inputs render the design of robust control systems an even harder endeavor. In this paper, we enhance a previously developed nonlinear adaptive flight control system [12], [13] by explicitly considering the presence of a state-dependent constraint for the input controlling the velocity loop, namely the fuel-to-air equivalence ratio (FER). This constraint is imposed by the very nature of the propulsion system, which is required to maintain the conditions that sustain scramjet operation. This is a very hard constraint for the system, which must be addressed by the guidance and control system to avoid choking of the scramjet engine. The recent paper [14] discusses issues related to the thermal choking of the engine which are relevant from the control design standpoint, and proposes the concept of

This work has been supported by AFRL under a subcontract from the Michigan/AFRL Collaborative Center in Control Science (MACCCS) and by DAGSI.

* Corresponding author, Department of Electrical and Computer Engineering, The Ohio State University, 2015 Neil Ave, Columbus, OH.
"FER margin" to quantify robustness with respect to the aforementioned constraint.

The approach we take here to address the presence of the hard constraint on the FER input is to integrate a novel adaptive guidance loop to a previously developed adaptive inner-loop control scheme. The role of the guidance system is to modify the bandwidth of a reference model to slow down the reference trajectory such that the control input limits are avoided, and to recover the desired speed of response whenever there is sufficient control authority to do so. To maintain the presentation streamlined, constraints on the deflection of the aerodynamic surfaces will not be explicitly addressed in this paper, although their presence can be easily incorporated in the scheme. In the literature, the presence of input constraints in adaptive control schemes has been addressed by resorting to direct modification of the update law or the reference model (see, for instance, the $\mu$-modification of [15] or the pseudo-control hedging of [16]. Here, we take a plug-in approach to the problem by leaving the adaptive tracking controller virtually unaltered, and shifting the burden of ensuring that the input constraints are not violated to an adaptive guidance system, integrated with the inner-loop controller. The methodology is inspired by the variable-bandwidth reference model employed in [17], and borrows a few ideas on self-optimizing controllers from [18]-[20]. With respect to this latter, the scheme presented here employs a novel adaptation law for the KuhnTucker multiplier in the overall cost function.

The paper is organized as follows: In Section II, the vehicle model employed in this study is briefly presented, and the state-dependent input constrained discussed. Section III presents the adaptive inner-loop controller, while Section IV is devoted to the adaptive guidance system. Simulation results are discussed in Section V, followed by concluding remarks.

\section{VEHICLE MODEL}

The control-oriented model of the longitudinal vehicle dynamics considered in this study is given by (see [4], [9])

$$
\begin{aligned}
\dot{V} & =\frac{T \cos \alpha-D}{m}-g \sin \gamma \\
\dot{h} & =V \sin \gamma \\
\dot{\gamma} & =\frac{L+T \sin \alpha}{m V}-\frac{g}{V} \cos \gamma \\
\dot{\theta} & =Q
\end{aligned}
$$




$$
\begin{aligned}
\dot{Q} & =\frac{M}{I_{y y}} \\
\dot{\eta} & =A_{\eta} \eta+N_{\eta}
\end{aligned}
$$

This model is comprised of five rigid-body state variables $x=[V, h, \gamma, \theta, Q]^{T}$, six aeroelastic states $\eta=$ $\left[\eta_{1}, \dot{\eta}_{1}, \ldots, \eta_{3}, \dot{\eta}_{3}\right]^{T}$ corresponding to the first three longitudinal bending modes, and three control inputs $u=$ $\left[\Phi, \delta_{c}, \delta_{e}\right]^{T}$ which affect (1) through the thrust, $T$, the pitching moment about the body $y$-axis, $M$, lift, $L$, and drag, $D$. The meaning of the state variables and the input vector is given in Table I. The output to be controlled is selected as $y=[V, h, \alpha]^{T}$. Following [9], approximations of the forces and moments to be employed for control design and stability analysis have been derived as follows:

$$
\begin{aligned}
& T \approx \bar{q} S\left[C_{T}^{\Phi}(\alpha) \Phi+C_{T}^{0}(\alpha)\right] \\
& L \cong \bar{q} S\left[C_{L}^{\alpha}(\alpha)+C_{L}^{\delta_{c}} \delta_{c}+C_{L}^{\delta_{e}} \delta_{e}+C_{L}^{\eta} \eta\right] \\
& D \approx \bar{q} S C_{D}^{\alpha}(\alpha) \\
& M \approx z_{T} T+\bar{q} \bar{c} S\left[C_{M}^{\alpha}(\alpha)+C_{M}^{\delta_{c}} \delta_{c}+C_{M}^{\delta_{e}} \delta_{e}+C_{M}^{\eta} \eta\right] \\
& N_{\eta} \approx \bar{q} S\left[N^{\alpha^{2}} \alpha^{2}+N^{\alpha} \alpha+N^{\delta_{c}} \delta_{c}+N^{\delta_{e}} \delta_{e}+N^{0}+N^{\eta} \eta\right]
\end{aligned}
$$

where

$$
\begin{aligned}
C_{T}^{\Phi}(\alpha) & =C_{T}^{\Phi \alpha^{3}} \alpha^{3}+C_{T}^{\Phi \alpha^{2}} \alpha^{2}+C_{T}^{\Phi \alpha} \alpha+C_{T}^{\Phi} \\
C_{T}(\alpha) & =C_{T}^{3} \alpha^{3}+C_{T}^{2} \alpha^{2}+C_{T}^{1} \alpha+C_{T}^{0} \\
C_{L}(\alpha) & =C_{L}^{\alpha} \alpha+C_{L}^{0} \\
C_{D}^{\alpha}(\alpha) & =C_{D}^{\alpha^{2}} \alpha^{2}+C_{D}^{\alpha} \alpha+C_{D}^{0} \\
C_{D}^{\delta}(\delta) & =C_{D}^{\delta_{c}^{2}} \delta_{c}^{2}+C_{D}^{\delta_{c}} \delta_{c}+C_{D}^{\delta_{e}^{2}} \delta_{e}^{2}+C_{D}^{\delta_{e}} \delta_{e} \\
C_{M}^{\alpha}(\alpha) & =C_{M}^{\alpha^{2}} \alpha^{2}+C_{M}^{\alpha} \alpha+C_{M}^{0}
\end{aligned}
$$

The relationship $\bar{q}=\frac{1}{2} V^{2} \rho_{0} \exp \left(-\left(h-h_{0}\right) / h_{s}\right)$ has been used for the dynamic pressure, where $\rho_{0}, h_{0}$ and $h_{s}$ are positive constants. It is assumed that all the coefficients of the control design model and all vehicle parameters are subject to uncertainty. The vector of all uncertain parameters is denoted by $\vartheta \in \mathbb{R}^{p}$ and it is assumed that $\vartheta \in \Theta$, where $\Theta$ is a known compact convex set. All model parameters will be considered constant during each tracking maneuver.

The input $\Phi$ is subject to a state-dependent constraint of the form $\Phi_{0}<\Phi<\Phi_{\mathrm{ch}}(\alpha, \bar{q}, V) \leq \Phi_{1}$, where $\Phi_{0}$ and $\Phi_{1}$ are respectively the constant upper and lower limits shown in Table I. The function $\Phi_{\mathrm{ch}}(\cdot)$ represents the maximum fuelto-air ratio that can be provided at a given flight condition compatible with scramjet combustion in the engine. As a matter of fact, heat addition (due to increased FER) tends to slow down the flow within the combustion chamber, until it becomes subsonic, at which point, scramjet combustion can no longer be sustained. While a closed-form solution for $\Phi_{\mathrm{ch}}(\cdot)$ can not be computed, an analytical expression can be given using curve-fitting. In this paper, a cubic spline was employed to derive an approximation of $\Phi_{\mathrm{ch}}(\cdot)$ over the range of interest, using the first-principle model in [4]. It is worth noting that $\Phi_{\mathrm{ch}}(\cdot)$ depends on the vehicle velocity, the angle-of-attack, and altitude (by way of the dynamic pressure), that is, on the regulated output. As an example,
TABLE I

ADMISSIBLE RANGES FOR STATE, INPUT, AND VARIABLES OF INTEREST

\begin{tabular}{c|c|cc} 
Variable & & Min Value & Max Value \\
\hline$V$ & Vehicle Velocity & $7500 \mathrm{ft} / \mathrm{s}$ & $11000 \mathrm{ft} / \mathrm{s}$ \\
$h$ & Vehicle Altitude & $85000 \mathrm{ft}$ & $135000 \mathrm{ft}$ \\
$\gamma$ & Flight-Path Angle (FPA) & $-3 \mathrm{deg}$ & $3 \mathrm{deg}$ \\
$\theta$ & Pitch Angle & $-5 \mathrm{deg}$ & $5 \mathrm{deg}$ \\
$Q$ & Pitch Rate & $-10 \mathrm{deg} / \mathrm{s}$ & $10 \mathrm{deg} / \mathrm{s}$ \\
\hline$\Phi$ & Fuel-to-air Ratio & 0.05 & 1.5 \\
$\delta_{c}$ & Canard Deflection & $-20 \mathrm{deg}$ & $20 \mathrm{deg}$ \\
$\delta_{e}$ & Elevator Deflection & $-20 \mathrm{deg}$ & $20 \mathrm{deg}$ \\
\hline$\alpha$ & Angle-of-Attack, $\alpha=\theta-\gamma$ & $-5 \mathrm{deg}$ & $5 \mathrm{deg}$ \\
$\bar{q}$ & Dynamic Pressure & $182.5 \mathrm{psf}$ & $2200 \mathrm{psf}$ \\
$\mathrm{M}$ & Mach Number & 6 & 12
\end{tabular}

Figure 1 shows plots of $\Phi_{\text {ch }}$ versus Mach number for given values of $\alpha$ at $\bar{q}=1800 \mathrm{psv}$. It can be noted that at lower Mach number the attainable thrust (hence, the vehicle acceleration) is limited by the choking conditions.

\section{Baseline Adaptive Controller}

In this section, we present the inner-loop adaptive controller developed in [13] that will be used in the integrated flight control scheme. The controller is developed ignoring the presence of the constraint on the control input, which, as mentioned, will be dealt with by the guidance system. The goal of the control algorithm is to steer the output of system (1) to a desired trim condition $y_{\infty}^{\star}=$ $\left[V_{\infty}^{\star}, h_{\infty}^{\star}, \alpha_{\infty}^{\star}\right]^{T}$, along smooth exogenous reference trajectories $y_{\text {ref }}(t)=\left[V_{\text {ref }}(t), h_{\text {ref }}(t), \alpha_{\text {ref }}(t)\right]^{T}$, robustly with respect to the considered model parameter uncertainty. Clearly, $\lim _{t \rightarrow \infty} y_{\text {ref }}(t)=y_{\infty}^{\star}$. The reference trajectories for the remaining state variables are defined as $\gamma_{\text {ref }}:=\dot{h}_{\text {ref }} / V_{\text {ref }}$, $\theta_{\text {ref }}:=\alpha_{\text {ref }}+\gamma_{\text {ref }}$ and $Q_{\text {ref }}:=\dot{\theta}_{\text {ref }}$, and let $x_{\text {ref }}=$ $\left[V_{\mathrm{ref}}, h_{\mathrm{ref}}, \gamma_{\mathrm{ref}}, \theta_{\mathrm{ref}}, Q_{\mathrm{ref}}\right]^{T}$. We say that $x_{\mathrm{ref}}$ is admissible if $x_{\text {ref }}(t) \in \mathcal{A}$, where $\mathcal{A}$ is the set defined by the admissible ranges given in Table I. For controller design, define com-

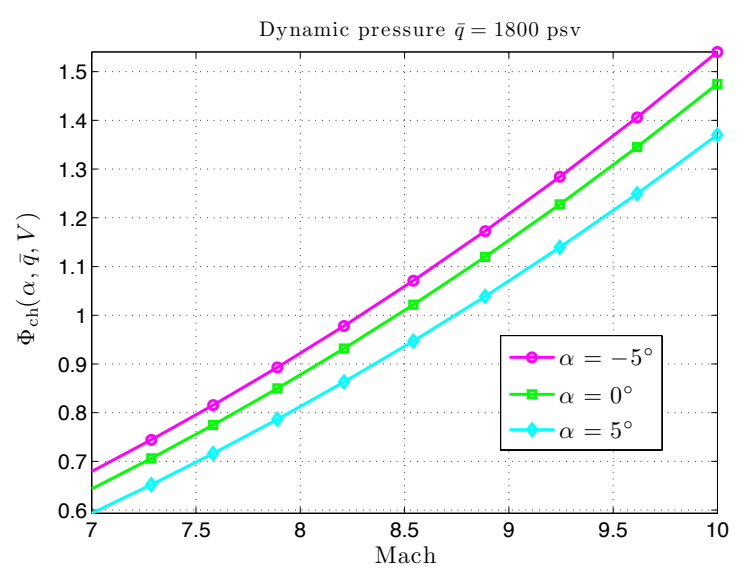

Fig. 1. Plot of $\Phi_{\mathrm{ch}}(\alpha, \bar{q}, V)$ vs. Mach number for different values of $\alpha$. 
manded trajectories as $\gamma_{\mathrm{cmd}}:=-k_{h} V_{\mathrm{ref}}\left[h-h_{\mathrm{ref}}\right] / V_{\mathrm{ref}}+\gamma_{\mathrm{ref}}$ and $Q_{\text {cmd }}:=-k_{\theta}\left[\theta-\theta_{\text {ref }}\right]+Q_{\text {ref }}$, where $k_{h}>0$ and $k_{\theta}>0$ are gain parameters, and let the tracking error be defined as $\tilde{x}=[\tilde{V}, \tilde{h}, \tilde{\gamma}, \tilde{\theta}, \tilde{Q}]^{T}:=\left[V-V_{\text {ref }}, h-h_{\text {ref }}, \gamma-\gamma_{\mathrm{cmd}}, \theta-\right.$ $\theta_{\text {ref }}, Q-Q_{\text {cmd }}$. Following [13], the plant model is written in the form

$$
\begin{aligned}
\dot{V} & =\vartheta_{1}^{T} \Psi_{1}(\bar{q}, \alpha)+\vartheta_{1}^{T} B_{1}(\bar{q}, \alpha) \Phi \\
\dot{h} & =V \sin \gamma \\
\dot{\gamma} & =\vartheta_{2}^{T} \Psi_{2}(x, \Phi)+\vartheta_{2}^{T} B_{2}(x) \delta+P_{2}(x) \eta \\
\dot{\theta} & =Q \\
\dot{Q} & =\vartheta_{3}^{T} \Psi_{3}(x, \Phi)+\vartheta_{3}^{T} B_{3}(x) \delta+P_{3}(x) \eta \\
\dot{\eta} & =A_{\eta} \eta+N_{\eta}(x, \eta, \delta)
\end{aligned}
$$

where $\vartheta_{i} \in \Theta_{i}, 1 \leq i \leq 3$, where $\Theta_{i} \subset \mathbb{R}^{p_{i}}$ are known compact convex sets. Accordingly, we define $\vartheta=$ $\operatorname{col}\left(\vartheta_{1}, \vartheta_{2}, \vartheta_{3}\right)$ and $\Theta=\Theta_{1} \times \Theta_{2} \times \Theta_{3}$. For notational convenience, let $\delta=\left[\delta_{c}, \delta_{e}\right]^{T}, \vartheta_{a}=\operatorname{col}\left(\theta_{2}, \theta_{3}\right)$ and $B_{a}\left(x, \theta_{a}\right)=$ $\operatorname{col}\left(\vartheta_{2}^{T} B_{2}(x), \vartheta_{3}^{T} B_{3}(x)\right)$. The certainty-equivalence adaptive controller considered in this study is given by the assignment

$$
\begin{gathered}
\Phi=\frac{1}{\hat{\vartheta}_{1}^{T} B_{1}(\alpha, \bar{q})}\left[-k_{V} \tilde{V}-\hat{\vartheta}_{1}^{T} \Psi_{1}(\alpha, \bar{q})+\dot{V}_{\mathrm{ref}}\right] \\
\delta=B_{a}^{-1}\left(x, \hat{\theta}_{a}\right)\left(\begin{array}{c}
-k_{\gamma} \tilde{\gamma}-\hat{\vartheta}_{2}^{T} \Psi_{2}(x, \Phi)+\dot{\gamma}_{\mathrm{cmd}} \\
-k_{Q} \tilde{Q}-\hat{\vartheta}_{3}^{T} \Psi_{3}(x, \Phi)+\dot{Q}_{\mathrm{cmd}}
\end{array}\right)
\end{gathered}
$$

together with the update laws

$$
\begin{aligned}
& \dot{\hat{\vartheta}}_{1}=\underset{\hat{\vartheta}_{1} \in \Theta_{1}}{\operatorname{Proj}}\left\{\Gamma_{1}\left[B_{1}(\alpha, \bar{q}) \Phi+\Psi_{1}(\alpha, \bar{q})\right] \tilde{V}+v_{1}\right\} \\
& \dot{\hat{\vartheta}}_{2}=\underset{\hat{\vartheta}_{2} \in \Theta_{2}}{\operatorname{Proj}}\left\{\Gamma_{2}\left[B_{2}(x) \delta+\Psi_{2}(x, \Phi)\right] \tilde{\gamma}+v_{2}\right\} \\
& \dot{\hat{\vartheta}}_{3}=\operatorname{Proj}_{\hat{\vartheta}_{3} \in \Theta_{3}}\left\{\Gamma_{3}\left[B_{3}(x) \delta+\Psi_{3}(x, \Phi)\right] \tilde{Q}\right\}
\end{aligned}
$$

where $k_{V}>0, k_{\gamma}>0$ and $k_{Q}>0$ are qain parameters, and $\Gamma_{i} \in \mathbb{R}^{p_{i} \times p_{i}}, 1 \leq i \leq 3$, are positive definite matrices. The external input $v=\left[v_{1}, v_{2}\right]^{T}$ will be used by the adaptive guidance law, yet to be defined. Setting this input to zero yields the baseline adaptive controller. Finally, let $\hat{\vartheta}=\operatorname{col}\left(\hat{\vartheta}_{1}, \hat{\vartheta}_{2}, \hat{\vartheta}_{3}\right)$. After the coordinate transformation $x \mapsto \tilde{x}$ and $\hat{\vartheta} \mapsto \tilde{\vartheta}:=\vartheta-\hat{\vartheta}$, the model (2) reads as ${ }^{1}$

$$
\begin{aligned}
\dot{\tilde{V}} & =-k_{V} \tilde{V}+\left[\Psi_{1}(\bar{q}, \alpha)+B_{1}(\bar{q}, \alpha) \Phi\right]^{T} \tilde{\vartheta}_{1} \\
\dot{\tilde{h}} & =-k_{h} \tilde{h}+V_{\mathrm{ref}} \tilde{\gamma}+\tilde{V} \sin \gamma \\
\dot{\tilde{\gamma}} & =-k_{\gamma} \tilde{\gamma}+\left[\Psi_{2}(x, \Phi)+B_{2}(x) \delta\right]^{T} \tilde{\vartheta}_{2}+P_{2}(x) \eta \\
\dot{\tilde{\theta}} & =-k_{\theta} \tilde{\theta}+\tilde{Q} \\
\dot{\tilde{Q}} & =-k_{Q} \tilde{Q}+\left[\Psi_{3}(x, \Phi)+B_{3}(x) \delta\right]^{T} \tilde{\vartheta}_{3}+P_{3}(x) \eta \\
\dot{\eta} & =A_{\eta} \eta+N_{\eta}(x, \eta, \delta)
\end{aligned}
$$

Using arguments similar to those in [13], it is possible to show that the baseline adaptive controller satisfies the following:

\footnotetext{
${ }^{1}$ In equation (5), whenever convenient, we have used the small-angle approximation $\sin \gamma \approx \gamma$.
}

Proposition 3.1: Assume that the control input is unconstrained. Fix, arbitrarily, compact sets of admissible initial conditions $\mathcal{K}_{x} \subset \mathcal{A}$ and $\mathcal{K}_{\eta}$ for the rigid-body and flexible states, respectively. Then there exists a selection of the controller gains $k_{V}, \ldots, k_{Q}$ and $\Gamma_{i}$ such that, for any admissible reference trajectory $x_{\text {ref }}(\cdot)$, all $(x(0), \eta(0), \hat{\vartheta}(0)) \in \mathcal{K}_{x} \times \mathcal{K}_{\eta} \times \Theta$, and for $v=0$, the trajectories of the closed-loop system (3)-(5) are bounded and satisfy $\lim _{t \rightarrow \infty} \tilde{x}(t)=0$.

In particular, in [13] it is shown that there exists a Lyapunov function of the form

$$
W(\tilde{x}, \tilde{\chi}, \tilde{\vartheta})=V(\tilde{x}, \tilde{\chi})+\tilde{\vartheta}^{T} \Gamma^{-1} \tilde{\vartheta}
$$

where $\eta \mapsto \tilde{\chi}$ is an invertible transformation involving the states of the flexible dynamics, $V(\tilde{x}, \chi)$ is a positive definite, radially unbounded and locally-quadratic function, and $\Gamma=$ $\operatorname{diag}\left(\Gamma_{1}, \Gamma_{2}, \Gamma_{3}\right)$, such that its derivative along trajectories of the closed-loop system (3)-(5) satisfies

$$
\dot{W}(\tilde{x}, \tilde{\chi}, \tilde{\vartheta}) \leq-\lambda_{1}\|\tilde{x}\|^{2}-\lambda_{2}\|\tilde{\chi}\|^{2}
$$

for all $(\tilde{x}, \tilde{\chi}) \in \Omega_{c}:=\{(\tilde{x}, \tilde{\chi}): V(\tilde{x}, \tilde{\chi}) \leq c\}$ and all $\hat{\vartheta} \in \Theta$, where $\lambda_{1}>0, \lambda_{2}>0$ are suitable constants, and $c>0$ is such that $\mathcal{K}_{x} \times \mathcal{K}_{\eta} \in \Omega_{c}$.

\section{AdAPtive GuidAnCE FOR The Velocity LoOP}

We turn now our attention to the design of the guidance outer loop to account for the constraint on the input $\Phi$. as mentioned, limits on the control surface deflection, $\delta$, will not be explicitly considered in this paper. Their incorporation on the guidance scheme via management of the reference $h_{\text {ref }}$ and $\alpha_{\text {ref }}$ can be accomplished in a straightforward manner by mimicking the development of guidance loop for $V_{\text {ref }}$.

First, define the feasible set $\mathcal{Y}$ for the output set-point as the set of all constant $y^{\star}=\left[V^{\star}, h^{\star}, \alpha^{\star}\right]^{T}$ for which the steady-state control input

$$
\Phi_{\mathrm{SS}}\left(\alpha^{\star}, \bar{q}^{\star}, \hat{\vartheta}_{1}\right)=-\frac{\hat{\vartheta}_{1}^{T} \Psi_{1}\left(\alpha^{\star}, \bar{q}^{\star}\right)}{\hat{\vartheta}_{1}^{T} B_{1}\left(\alpha^{\star}, \bar{q}^{\star}\right)}
$$

is such that

$$
\Phi_{0}<\Phi_{\mathrm{Ss}}\left(\alpha^{\star}, \bar{q}^{\star}, \hat{\vartheta}_{1}\right)<\Phi_{\mathrm{ch}}\left(\alpha^{\star}, \bar{q}^{\star}, V^{\star}\right)
$$

for all $\hat{\vartheta}_{1} \in \Theta_{1}$. Let $y_{0}^{\star}$ and $y_{\infty}^{\star}$ define respectively feasible values of the initial and the desired setpoints. The adaptive guidance system for the velocity loop is given by

$$
\begin{aligned}
\dot{V}_{\text {ref }} & =-\kappa V_{\text {ref }}+\kappa V_{\infty}^{\star} \quad V_{\text {ref }}(0)=V_{0}^{\star} \\
\dot{\kappa} & =\tau\left(x, x_{\text {ref }}, \hat{\vartheta}\right)
\end{aligned}
$$

where $\tau(\cdot)$ is an update law to be defined. For the sake of simplicity, the reference trajectory $\alpha_{\text {ref }}(t)$ is obtained by filtering through a second-order stable LTI filter a step commands of amplitude $\alpha_{\infty}^{\star}-\alpha_{0}^{\star}$. The reference trajectory $h_{\text {ref }}$ is obtained from the expression of $\bar{q}$ so as to maintain a constant desired dynamic pressure $\bar{q}^{\star}$, that is,

$$
h_{\text {ref }}(t)=h_{0}+h_{s}\left[\ln \left(\rho_{0} V_{\text {ref }}^{2}(t)\right)-\ln \left(2 \bar{q}^{\star}\right)\right]
$$


Let $c\left(\Phi, \Phi_{\mathrm{ch}}\right)$ be defined as

$$
c\left(\Phi, \Phi_{\mathrm{ch}}\right)=\left(\begin{array}{c}
\Phi_{0}-\Phi\left(\alpha, \bar{q}, \dot{V}_{\mathrm{ref}}, \hat{\vartheta}\right) \\
\Phi\left(\alpha, \bar{q}, \dot{V}_{\mathrm{ref}}, \hat{\vartheta}\right)-\Phi_{\mathrm{ch}}(\alpha, \bar{q}, V)
\end{array}\right)
$$

so that the feasibility condition for the velocity guidance loop is given by $c_{i}\left(\Phi, \Phi_{\mathrm{ch}}\right)<0, i=1,2$. Define the logarithmic barrier function

$$
b\left(\Phi, \Phi_{\mathrm{ch}}\right):=b_{0}-\ln \left(-c_{1}\right)-\ln \left(-c_{2}\right)
$$

where $b_{0}>0$ is selected such that $b(\cdot) \geq \bar{b}_{0}>0$ for all $\Phi_{0}<$ $\Phi<\Phi_{\text {ch }}$. Note that $b\left(\Phi, \Phi_{\text {ch }}\right)$ is indeed a function of the variables $\alpha, \bar{q}, \tilde{V}, V_{\text {ref }}, \dot{V}_{\text {ref }}, \hat{\vartheta}_{1}$. Consider the cost function

$$
J\left(\kappa, \varrho, \Phi, \Phi_{\mathrm{ch}}\right):=\frac{1}{2}\left(\kappa-\kappa^{\star}\right)^{2}+\varrho b\left(\Phi, \Phi_{\mathrm{ch}}\right)+\frac{1}{3} \varrho^{3}
$$

where $\rho \in \mathbb{R}_{\geq 0}$ is a Kuhn-Tucker multiplier and $\kappa^{\star}>0$ is the desired value of the gain of the adaptive filter (6). Since $b(\cdot)>0$, for any fixed $\Phi \in\left(\Phi_{0}, \Phi_{\text {ch }}\right)$ the function $J(\cdot)$ is a positive definite function of $\left(\kappa-\kappa^{\star}, \varrho\right)$ over the set $\mathcal{X}=\mathbb{R} \times \mathbb{R}_{\geq 0} ;$ moreover, for any $(\kappa, \varrho) \in \mathcal{X}-\left(\kappa^{\star}, 0\right)$,

$$
\lim _{\Phi \rightarrow \Phi_{0}^{+}} J(\cdot)=+\infty, \quad \lim _{\Phi \rightarrow \Phi_{\text {ch }}^{-}} J(\cdot)=+\infty
$$

The derivative of the cost function $J(\cdot)$ along trajectories of the closed-loop system reads as

$$
\dot{J}=\left(\kappa-\kappa^{\star}\right) \dot{\kappa}+\left[\varrho^{2}+b\left(\Phi, \Phi_{\mathrm{ch}}\right)\right] \dot{\varrho}+\varrho \dot{b}\left(\Phi, \Phi_{\mathrm{ch}}\right)
$$

Since

$$
\begin{gathered}
\dot{\Phi}=\frac{\partial \Phi}{\partial \alpha} \dot{\alpha}+\frac{\partial \Phi}{\partial \bar{q}}\left[\frac{\partial \bar{q}}{\partial h} \dot{h}+\frac{\partial \bar{q}}{\partial V} \dot{V}\right]+\frac{\partial \Phi}{\partial \tilde{V}}\left[\dot{V}+\dot{V}_{\text {ref }}\right] \\
+\frac{\partial \Phi}{\partial \dot{V}_{\text {ref }}} \ddot{V}_{\text {ref }}+\frac{\partial \Phi}{\partial \hat{\vartheta}_{1}} \dot{\hat{\vartheta}}_{1} \\
\dot{\Phi}_{\text {ch }}=\frac{\partial \Phi_{\text {ch }}}{\partial \alpha} \dot{\alpha}+\frac{\partial \Phi_{\text {ch }}}{\partial \bar{q}}\left[\frac{\partial \bar{q}}{\partial h} \dot{h}+\frac{\partial \bar{q}}{\partial V} \dot{V}\right]+\frac{\partial \Phi_{\text {ch }}}{\partial V} \dot{V}
\end{gathered}
$$

one obtains

$$
\begin{aligned}
\dot{b}= & {\left[\frac{\partial b}{\partial \Phi} \frac{\partial \Phi}{\partial \alpha}+\frac{\partial b}{\partial \Phi_{\mathrm{ch}}} \frac{\partial \Phi_{\mathrm{ch}}}{\partial \alpha}\right]\left[Q-\vartheta_{2}^{T} \Psi_{2}(x, \Phi)-\vartheta_{2}^{T} B_{2}(x) \delta\right] } \\
& +\left[\frac{\partial b}{\partial \Phi} \frac{\partial \Phi}{\partial \bar{q}}+\frac{\partial b}{\partial \Phi_{\mathrm{ch}}} \frac{\partial \Phi_{\mathrm{ch}}}{\partial \bar{q}}\right] \frac{\partial \bar{q}}{\partial h} V \sin \gamma \\
& +\left[\frac{\partial b}{\partial \Phi}\left[\frac{\partial \Phi}{\partial \bar{q}} \frac{\partial \bar{q}}{\partial V}+\frac{\partial \Phi}{\partial \tilde{V}}\right]+\frac{\partial b}{\partial \Phi_{\mathrm{ch}}}\left[\frac{\partial \Phi_{\mathrm{ch}}}{\partial \bar{q}} \frac{\partial \bar{q}}{\partial V}+\frac{\partial \Phi_{\mathrm{ch}}}{\partial V}\right]\right] \\
& \times\left[\vartheta_{1}^{T} \Psi_{1}(\bar{q}, \alpha)+\vartheta_{1}^{T} B_{1}(\bar{q}, \alpha) \Phi\right]+\frac{\partial b}{\partial \Phi}\left[\frac{\partial \Phi}{\partial \tilde{V}} \dot{V}_{\mathrm{ref}}+\frac{\partial \Phi}{\partial \hat{\vartheta}_{1}} \dot{\hat{\vartheta}}_{1}\right] \\
& +\frac{\partial b}{\partial \Phi} \frac{\partial \Phi}{\partial \dot{V}_{\text {ref }}}\left[\kappa^{2} V_{\mathrm{ref}}-\kappa^{2} V_{\infty}^{\star}-V_{\text {ref }} \dot{\kappa}\right]
\end{aligned}
$$

Collecting terms, the derivative of the barrier function along solutions of the closed-loop system can be written as

$$
\begin{aligned}
\dot{b}= & l_{0}\left(x, x_{\mathrm{ref}}, \hat{\vartheta}, v\right)+l_{1}^{T}\left(x, x_{\mathrm{ref}}, \hat{\vartheta}\right) \vartheta_{1}+l_{2}^{T}\left(x, x_{\mathrm{ref}}, \hat{\vartheta}\right) \vartheta_{2} \\
& +l_{\kappa}\left(x, x_{\mathrm{ref}}, \hat{\vartheta}\right) \dot{\kappa}
\end{aligned}
$$

Combining (7) with (8), the derivative of the cost function reads as

$$
\begin{aligned}
\dot{J}= & {\left[\kappa-\kappa^{\star}+\varrho l_{\kappa}\left(x, x_{\mathrm{ref}}, \hat{\vartheta}\right)\right] \dot{\kappa}+\left[\varrho^{2}+b\left(\Phi, \Phi_{\mathrm{ch}}\right)\right] \dot{\varrho} } \\
& +l_{0}\left(x, x_{\mathrm{ref}}, \hat{\vartheta}, v\right)+\varrho l_{1}^{T}\left(x, x_{\mathrm{ref}}, \hat{\vartheta}\right) \vartheta_{1} \\
& +\varrho l_{2}^{T}\left(x, x_{\mathrm{ref}}, \hat{\vartheta}\right) \vartheta_{2}
\end{aligned}
$$

The update law for $\kappa$ and $\varrho$ are chosen respectively as

$$
\begin{aligned}
\dot{\kappa}= & -\epsilon_{1}\left[\kappa-\kappa^{\star}+\varrho l_{\kappa}\left(x, x_{\mathrm{ref}}, \hat{\vartheta}\right)\right] \\
\dot{\varrho}= & \frac{1}{\varrho^{2}+b\left(\Phi, \Phi_{\mathrm{ch}}\right)}\left[-\epsilon_{2} \varrho^{2}-l_{0}\left(x, x_{\mathrm{ref}}, \hat{\vartheta}, v\right)\right. \\
& \left.-\varrho l_{1}^{T}\left(x, x_{\mathrm{ref}}, \hat{\vartheta}\right) \hat{\vartheta}_{1}-\varrho l_{2}^{T}\left(x, x_{\mathrm{ref}}, \hat{\vartheta}\right) \hat{\vartheta}_{2}\right]
\end{aligned}
$$

where $\epsilon_{1}>0$ and $\epsilon_{2}>0$ are design parameters, with initial conditions $\kappa(0)=\kappa_{0} \geq 0$ and $\varrho(0)=\varrho_{0}>0$. The additional input $v$ in the update law (4) for the parameters of the innerloop controller is selected as

$$
v_{1}=\Gamma_{1} l_{1}\left(x, x_{\mathrm{ref}}, \hat{\vartheta}\right) \varrho, \quad v_{2}=\Gamma_{2} l_{2}\left(x, x_{\mathrm{ref}}, \hat{\vartheta}\right) \varrho
$$

Note that, since $b\left(\Phi, \Phi_{\text {ch }}\right) \geq \bar{b}_{0}>0$ in its domain of definition, the update law for $\varrho$ is well-defined.

Proposition 4.1: Consider the closed-loop system given by the plant model (2), the inner-loop controller (3)-(4), and the adaptive guidance system (6), (10)-(11). Assume that the initial setpoint $y_{0}^{\star}$ and the desired setpoint $y_{\infty}^{\star}$ are feasible. Then, for any feasible initial condition $\left(x_{0}, \eta_{0}\right)$, the trajectories of the closed-loop system are bounded and satisfy $\lim _{t \rightarrow \infty} \tilde{x}(t)=0$. Moreover, the input $\Phi$ satisfies $\Phi_{0}<\Phi(t)<\Phi_{\operatorname{ch}}(\alpha(t), \bar{q}(t), V(t))$ for all $t \in[0, \infty)$, and $\lim _{t \rightarrow \infty} V_{\text {ref }}(t)=V_{\infty}^{\star}, \lim _{t \rightarrow \infty} \kappa(t)=\kappa^{\star}$.

Proof: Consider the Lyapunov function candidate

$$
\mathcal{W}(\tilde{x}, \tilde{\chi}, \tilde{\vartheta}, \kappa, \varrho)=W(\tilde{x}, \tilde{\chi}, \tilde{\vartheta})+J\left(\kappa, \varrho, \Phi, \Phi_{\mathrm{ch}}\right)
$$

which is positive definite in $\left(\tilde{x}, \tilde{\chi}, \tilde{\vartheta}, \kappa-\kappa^{\star}, \varrho\right) \in \Omega_{c} \times \mathbb{R}^{p} \times$ $\mathcal{X}$, and evaluate its derivative along trajectories of the closedloop system to obtain

$$
\begin{gathered}
\dot{\mathcal{W}} \leq- \\
-\lambda_{1}\|\tilde{x}\|^{2}-\lambda_{2}\|\tilde{\chi}\|^{2}-\epsilon_{1}\left[\kappa-\varrho^{\star}+\varrho l_{\kappa}\left(x, x_{\mathrm{ref}}, \hat{\vartheta}\right)\right]^{2}
\end{gathered}
$$

where we have used the properties of the smooth parameter projection. Since by assumption $\varrho_{0}>0$, and $\varrho=0$ is an equilibrium for the dynamics of $\varrho$ given by the second equation in (10), it follows that $\varrho(t)>0$ for all $t \geq 0$. As a result, for any feasible initial condition, there exist $d>0$ such that $(\tilde{x}(t), \tilde{\chi}(t), \tilde{\vartheta}(t), \kappa(t), \varrho(t)) \in \Omega_{d}^{\mathcal{W}}:=\{\mathcal{W} \leq d\}$ for all $t \geq 0$, where $\Omega_{d}^{\mathcal{W}}$ is compact. Note also that, since by construction $\mathcal{W}$ is proper on the set $\left\{\left(x, \hat{\vartheta}, x_{\text {ref }}\right): \Phi \in\right.$ $\left.\left(\Phi_{0}, \Phi_{\mathrm{ch}}\right)\right\}$, and the trajectories of the closed-loop system evolve in a compact set, necessarily $\Phi(t)$ remains bounded away from its lower and upper limits. By LaSalle's invariance principle, trajectories converge to the largest invariant set contained in the set

$$
\mathcal{S}:=\left\{\tilde{x}=0, \tilde{\chi}=0, \kappa-\kappa^{\star}=0, \varrho=0\right\}
$$

from which the result follows.

From the result of Proposition 4.1 it is evident that the guidance system modifies adaptively the rate of change of the velocity reference in such a way that the input constraints are avoided during the transient, while recovering both the desired value of the bandwidth of the pre-filter (basically, $\kappa^{\star}$ ) and the desired set-point at steady-state, $V_{\infty}^{\star}$. The fact that the Kuhn-Tucker multiplier vanishes at stead-state is expected, as the desired set-point is assumed feasible. 

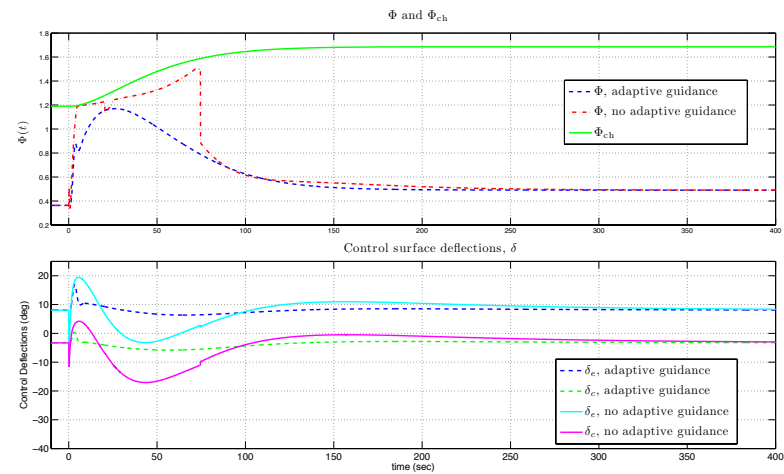

Fig. 2. Control inputs with and without adaptive guidance. Top plot: input $\Phi(t)$ and $\Phi_{\mathrm{ch}}(t)$; bottom plot: input $\delta(t)$.

\section{Simulation Results}

The proposed scheme has been tested in simulation using the nonlinear control-oriented model (1). The maneuver considered in the simulation presented here is a climbing at constant dynamic pressure $\bar{q}=1800$ psv, obtained by increasing the velocity from an initial trim condition at $V_{0}^{\star}=9000 \mathrm{tf} / \mathrm{s}$ to a final trim at $V_{\infty}^{\star}=10500 \mathrm{ft} / \mathrm{s}$. This correspond to climbing from approximately $h_{0}^{\star} \approx 9.4 \times 10^{4} \mathrm{ft}$ to $h_{\infty}^{\star} \approx 10.05 \times 10^{4} \mathrm{ft}$. The angle-of-attack is kept constant at $\alpha^{\star}=2 \mathrm{deg}$. The parameter of the adaptive inner-loop controller have been selected as in [13], whereas the parameters of the guidance law have been selected as follows:

$$
\begin{aligned}
& \epsilon_{1}=0.1 \quad \epsilon_{2}=0.05 \\
& \kappa_{0}=0 \quad \varrho_{0}=1 \\
& \kappa^{\star}=75
\end{aligned}
$$

For the first simulation, only static saturations corresponding to the values on Table I have been incorporated in the controlled system, and the adaptive guidance law has been removed. In this case, the gain of the pre-filter for the velocity reference has been kept fixed at $\kappa=75$. The result of this simulation, as far as the input is concerned, is visible in the top plot of Figure 2 (red dash-dotted line) showing $\Phi(t)$, and the bottom plot of the same figure (solid cyan and magenta lines) showing $\delta(t)$. The control effort for $\Phi(t)$ reaches the choking condition (green solid line) during the first few seconds of the simulation after the reference command is issued at $t=0$. The simulation should have terminated when the choking condition is met, as the conditions that sustain scramjet propulsion are violated. On the other hand, when the adaptive guidance is active, the control input $\Phi$ is prevented from reaching $\Phi_{\text {ch }}$ during the initial transient (blue dashed line in the top plot of Figure 2). The surface deflections remain also very well-behaved as seen in the bottom plot of Figure 2 (dashed blue and green lines). Figure 3 compares the vehicle velocity $V(t)$ under the adaptive guidance and control systems with the reference $V_{\text {ref }}$, computed with and without adaptive guidance. It can be seen that the three trajectories are almost identical, and differ

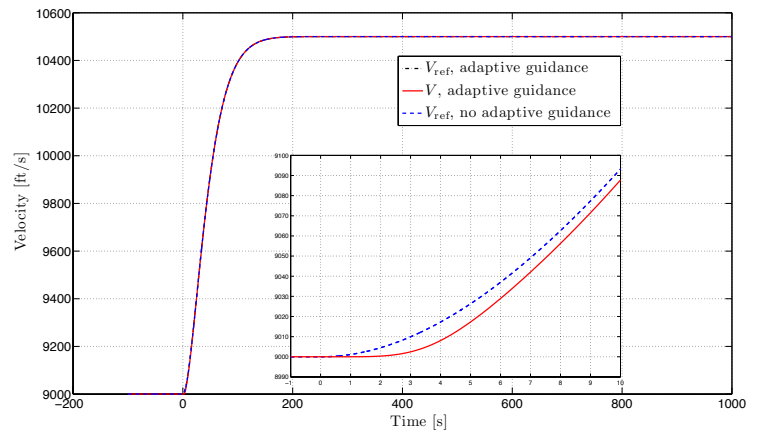

Fig. 3. Vehicle velocity, $V(t)$, and velocity reference, $V_{\text {ref }}(t)$, with and without adaptive guidance.
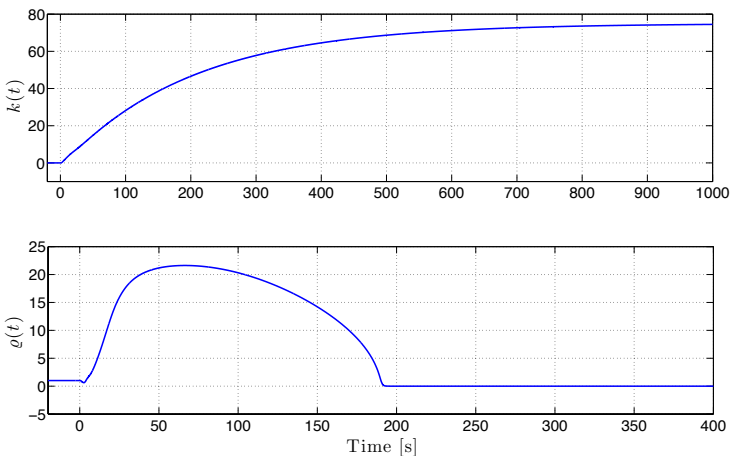

Fig. 4. Parameters of the adaptive guidance scheme, $\kappa(t)$ and $\varrho(t)$.

only slightly during the first few seconds after the command is issued. Finally, Figure 4 shows $\kappa(t)$ and $\varrho(t)$, respectively. As expected, $\varrho(t)$ is active only when the constraint would be violated, and settles to zero very rapidly after $t=200 \mathrm{~s}$, whereas the gain $\kappa(t)$ converges to the desired steady-state value.

\section{CONCLUSION}

The adaptive guidance and control scheme presented in this paper constitutes a preliminary step towards the definition of a truly integrated flight control architecture for hypersonic vehicles. Current work is addressing the incorporation of rate-limits and constraints on the aerodynamic control surface, as well as the adoption of anti-windup schemes to alleviate the burden on the guidance system.

\section{REFERENCES}

[1] F. R. Chavez and D. K. Schmidt, "Analytical aeropropulsive/aeroelastic hypersonic-vehicle model with dynamic analysis," Journal of Guidance, Control, and Dynamics, vol. 17, no. 6, pp. 1308-19, 1994.

[2] K. Bilimoria and D. Schmidt, "Integrated development of the equations of motion for elastic hypersonic flight vehicles," Journal of Guidance, Control, and Dynamics, vol. 18, no. 1, pp. 73-81, 1995.

[3] M. A. Bolender and D. B. Doman, "Flight path angle dynamics of airbreathing hypersonic vehicles," in Guidance, Navigation, and Control Conference and Exhibit, Keystone, CO, 2006, AIAA Paper 2006-6692. 
[4] _ " "A nonlinear longitudinal dynamical model of an air-breathing hypersonic vehicle," Journal of Spacecraft and Rockets, vol. 44, no. 2 , pp. 374-387, 2007.

[5] M. Bolender and D. Doman, "Modeling unsteady heating effects on the structural dynamics of a hypersonic vehicle," in AIAA Atmospheric Flight Mechanics Conference and Exhibit, Keystone, CO, 2006, AIAA Paper 2006-6646.

[6] M. Oppenheimer and D. Doman, "A hypersonic vehicle model developed with piston theory," in AIAA Atmospheric Flight Mechanics Conference and Exhibit, Keystone, CO, 2006, AIAA Paper 2006-6637.

[7] R. Lind, "Linear parameter-varying modeling and control of structural dynamics with aerothermoelastic effects," Journal of Guidance, Control, and Dynamics, vol. 25, no. 4, pp. 733-9, 2002.

[8] B. Fidan, M. Mirmirani, and P. Ioannou, "Flight dynamics and control of air-breathing hypersonic vehicles: Review and new directions," in 12th AIAA International Space Planes and Hypersonic Systems and Technologies, Norfolk,VA, 2003, AIAA Paper 2003-7081.

[9] J. T. Parker, A. Serrani, S. Yurkovich, M. A. Bolender, and D. B. Doman, "Control-oriented modeling of an air-breathing hypersonic vehicle," Journal of Guidance, Control, and Dynamics, vol. 30, no. 3, pp. 856-869, 2007.

[10] D. Sigthorsson, P. Jankovsky, A. Serrani, S. Yurkovich, M. Bolender, and D. Doman, "Robust linear output feedback control of an airbreathing hypersonic vehicle," Journal of Guidance Control and Dynamics, vol. 31, no. 4, pp. 1052-1066, 2008.

[11] L. Fiorentini, A. Serrani, M. A. Bolender, and D. B. Doman, "Robust nonlinear sequential loop closure control design for an air-breathing hypersonic vehicle model," in Proceedings of the 2008 American Control Conference, Seattle, WA, 2008.

[12] — "Nonlinear control of a hypersonic vehicle with structural flexibility," in Proceedings of the 47th IEEE Conference on Decision and Control, Cancun, MX, 2008.

[13] L. Fiorentini, A. Serrani, M. Bolender, and D. Doman, "Nonlinear robust adaptive control of flexible air-breathing hypersonic vehicles," Journal of Guidance, Control and Dynamics, vol. 32, no. 2, pp. 402417, March-April 2009.

[14] A. Rodriguez, J. Dickeson, O. Cifdaloz, A. Kelkar, J. Vogel, D. Soloway, J. Benavides, and S. Sridharan, "Modeling and control of scramjet-powered hypersonic vehicles: Challenges, trends, and tradeoffs," in AIAA Guidance, Navigation and Control Conference and Exhibit, Honolulu, Hawaii, 2008, AIAA Paper 2008-6793.

[15] E. L. N. Hovakimyan, "Stable adaptation in the presence of actuator constraints with flight control applications," Journal of Guidance, Control, and Dynamics, vol. 30, no. 2, pp. 337-345, 2007.

[16] E. Jonhson and A. Calise, "Limited authority adaptive flight control for reusable launch vehicles," Journal of Guidance, Control, and Dynamics, vol. 26, no. 6, pp. 906-913, 2003.

[17] J. Schierman, D. Ward, J. Hull, N. Gandhi, M. Oppenheimer, and D. Doman, "Integrated adaptive guidance and control for re-entry vehicles with flight-test results," Journal of Guidance, Control, and Dynamics, vol. 27, no. 6, pp. 975-88, 2004.

[18] T. Johansen, "Optimizing nonlinear control allocation," in Proceedings of the 43rd IEEE Conference on Decision and Control, Paradise Island, Bahamas, 2004, pp. 3435-3440.

[19] J. Tjønnas and T. Johansen, "Adaptive optimizing nonlinear control allocation," in Proc. of the 16th IFAC World Congress, 2005.

[20] T. Johansen and D. Sbarbaro, "Lyapunov-based optimizing control of nonlinear blending processes," IEEE Transactions on Control Systems Technology, vol. 13, no. 4, pp. 631-638, 2005 